\title{
Osteoporotic Hip Fractures in the Elderly: Is There a Gender Difference?
}

\section{Sharifah Aishah Wan}

Sarawak General Hospital

Ing Khieng Tiong

Sarawak General Hospital

Seow Lin Chuah

Sarawak General Hospital

Yaw Kiet Cheong

Sarawak General Hospital

Benjamin Sachdev Manjit Singh

Sarawak General Hospital

Kar Hoo Lee

Sarawak General Hospital

Wendy Wan Hui Lee

Sarawak General Hospital

Cheng Lay Teh

Sarawak General Hospital

Jeh Kiong Tiong

Sarawak General Hospital

Affizal Samsudin

Universiti Malaysia Sarawak

Ahmad Tirmizi Jobli ( $\Delta$ jatirmizi@unimas.my)

Universiti Malaysia Sarawak

\section{Research Article}

Keywords: Osteoporosis, hip fracture, secondary osteoporosis, gender difference

Posted Date: November 9th, 2021

DOI: https://doi.org/10.21203/rs.3.rs-902613/v2

License: (1) (i) This work is licensed under a Creative Commons Attribution 4.0 International License. Read Full License 


\section{Abstract}

\section{Background}

Osteoporosis and osteoporotic fracture pose a major public health problem in our aging population and carry an increased morbidity and mortality. While overall diagnosis and treatment for osteoporosis have improved, osteoporosis in men remain underdiagnosed and undertreated.

\section{Aim}

We aim to describe the difference in clinical characteristics between elderly men and women with osteoporotic hip fractures in Sarawak General Hospital.

\section{Methods}

All patients diagnosed with osteoporotic hip fracture admitted from June 2019-March 2021 were recruited and demographic data and clinical features were obtained.

\section{Results}

There were 140 patients with osteoporotic hip fracture, and 40 were men (28.6\%). The mean age for males were $74.1 \pm 9.5$ years, while the mean age for females were $77.4 \pm 9.1$ years $(p=0.06)$. There were 20 men with secondary osteoporosis (50\%), while 13 women (13\%) had secondary osteoporosis $(p<0.001)$. The causes of secondary osteoporosis among the men were hypogonadism, COPD, GIOP, renal disease, ADT, thyroid disorder, prostate cancer, and previous gastrectomy. There were two deaths among the men and four deaths among the women during the inpatient and 3 months follow up period. There was no statistical significance between the mortality rates between male patients $(5 \%)$ and female patients $(4 \%)(p=0.55)$.

\section{Conclusion}

There were more females with osteoporotic hip fractures, and there were significantly more males with secondary osteoporotic hip fractures.

Trial registration: This study (Male osteoporotic hip fracture in Sarawak General Hospital) was registered with the Malaysian National Medical Research Register (NMRR). Trial registration number: NMRR-19-32346068 IIR

\section{Background}

Osteoporosis is an important public health problem globally, particularly in our aging population. Osteoporotic hip fractures will also cause increased economic burden on healthcare costs. Cheung et al projected the number of hip fracture in Asia will increase from 1,124,060 in 2018 to 2,563,488 in 2050, a 
2.28-fold increase. The direct cost of hip fracture will increase from 9.5 billion USD in 2018 to 15 billion USD in 2050, a 1.59-fold increase ${ }^{1}$.

While awareness of post-menopausal osteoporosis in women is increasing, along with developments in the management of the condition, male osteoporosis continues to be under-recognized and under-treated. There are differences between male and female osteoporosis. The incidence of osteoporotic fractures in both men and women increases with aging, however in men the osteoporotic fractures happened about 10 years later than women ${ }^{2}$. The prevalence of osteoporosis in United States (US) men >50 years old was $3-6 \%$ whereas in women $>50$ years old it was $13-18 \%^{3}$. The biggest impact of osteoporosis is obviously the incidence of osteoporotic fractures, especially spinal and hip fractures. Hip fractures carry a high morbidity and mortality. For men, the incidence of hip fractures in the US ranged from 0.56 per 1000 patients per year at age 60 years to 13 per 1000 patients per year by age 85 years ${ }^{4}$.

Even though the prevalence of osteoporosis and osteoporotic hip fractures is higher in women, men seem to have a worse outcome and mortality after a hip fracture ${ }^{5}$. Haentjens et al studied the excess mortality after a hip fracture in older men and women and concluded that older adults have a 5-8-fold increased risk for all-cause mortality during the first 3 months after a hip fracture. Excess annual mortality persisted over time for both women and men, but at any given age, excess annual mortality after hip fracture was higher in men than in women ${ }^{6}$. It is thought that the reason for the higher mortality in men after a hip fracture was due to men having higher co-morbid conditions, men being older and more post-operative complications. Simunovic et al reviewed the risk of death and post-operative complications among patients with hip fractures in 5 studies. Hip fractures were associated with 14-36\% one year mortality rate. They also found that earlier surgery significantly reduced the risk of mortality ${ }^{7}$. Within one year of hip fracture, $50 \%$ of men required institutionalization and only $20 \%$ return to their pre-fracture level of independence ${ }^{8}$.

There is a gradual decline in testosterone with aging in men, as opposed to an abrupt loss of oestrogen in menopausal women. This results in the higher bone loss in menopausal women, and subsequently increased incidence of osteoporotic fractures in menopausal women, and men experiencing osteoporotic fractures almost 10 years later than women. Bone loss increases with advancing age in men, as was demonstrated by an increase in average bone mineral density (BMD) loss at the femoral neck with increasing age in the Osteoporotic Fractures in Men (MrOs) study ${ }^{9}$. The authors of the MrOs also showed that lower BMD is associated with higher fracture risk in men; each standard deviation (SD) decrease in hip BMD increased the risk of hip fracture by $3.2-$ fold $^{9}$. The age-adjusted annual rate of hip and nonvertebral fracture was 2.4 and 14 per 1000 person-years respectively, on 4.4 years of follow up ${ }^{9}$. The risk factors for low BMD and fractures in men are low weight, low physical activity, medications (selective serotonin reuptake inhibitors (SSRI), anti-epileptic drugs (AEDs)), and medical conditions (abdominal aortic aneurysm, Parkinson's disease, poor renal function, chronic obstructive pulmonary disease, diabetes mellitus, diffuse idiopathic skeletal hyperostosis (DISH), certain ethnicity, vitamin D deficiency and hyperparathyroidism). 
Secondary osteoporosis may be present in both men and women, but some studies stated that secondary osteoporosis were more common in men than women ${ }^{2}$. Identification of causes of secondary osteoporosis is useful as treatment of the underlying condition will usually help with the treatment of osteoporosis as well ${ }^{8}$. The most common causes of secondary osteoporosis are glucocorticoid excess, hypogonadism and excessive alcohol consumption. Other causes are gastrointestinal malabsorption syndrome, renal insufficiency, chronic respiratory disorders, rheumatoid arthritis, malignancy, anaemia, hyperthyroidism or excess thyroxine, hyperparathyroidism, anticonvulsants, smoking and immobilization ${ }^{10-11}$. Therefore, a careful history, physical examination and appropriate blood investigation (such as a full blood count, renal profile, liver profile, calcium, thyroid-stimulating hormone (TSH), 25-hydroxyvitamin D and testosterone) is needed during an evaluation of patients with osteoporotic risk factors and/or osteoporotic fractures.

The Malaysian hip fracture national registry in 2008-2009 from selected government healthcare facilities reported 510 cases of hip fractures and 165 were men $(32 \%)^{12}$. Trivial falls were the main mechanism of hip fractures ${ }^{12-14}$. The risk factors associated with osteoporosis were advanced age, certain ethnicity, the female gender, family history, low body mass index, sedentary lifestyle, smoking, alcohol, low calcium and vitamin $D$ intake ${ }^{15}$. Malaysia would expect to have increased incidence of male osteoporotic fractures as life expectancy improves. Increased awareness about this condition will hopefully result in improved screening, diagnosis and treatment of the condition.

The objective of this study was to describe the gender difference in clinical characteristics in elderly patients with osteoporotic hip fractures in Sarawak General Hospital.

\section{Methods}

This was a prospective, observational study. This study, titled 'Male osteoporotic hip fractures in Sarawak General Hospital' was registered in the Malaysian National Medical Research Register (NMRR) and received approval from the Malaysian Medical Research and Ethics Committee (MREC) (NMRR-19-32346068 IIR) and was performed in accordance with the Declaration of Helsinki. All patients with osteoporotic hip fractures admitted to the Orthopaedics Ward, Sarawak General Hospital from June 2019 until March 2021 were recruited into the study after giving informed consent. Data regarding demographics, type of fractures, surgery, health co-morbidities, investigation results, dual energy $\mathrm{x}$ ray absorptiometry (DXA), and treatments were collected. All patients were assessed for causes of secondary osteoporosis clinically and additional blood investigations as necessary. All treatments for osteoporosis were according to the standard of care. The patients were subsequently followed up in the osteoporosis or geriatric clinics 3 months later and further clinical data were collected among those who attended the clinics, including the death outcome. The sample size was calculated based on the expected frequency of male osteoporotic hip fracture at $10 \%$ with a margin of error at $5 \%$. The minimum required sample size was 138 based on a $95 \%$ confidence interval. This is quoted using Epi Info software by CDC. 
Statistical analysis was performed using IBM SPSS Statistics version 25 software. Descriptive data is expressed as mean \pm SD. ANOVA is used for comparison of means between groups. Categorical data are presented as frequency and percentage and analysed using Chi-square or Fisher's exact test. A value of $p$ $<0.05$ is considered statistically significant.

\section{Results}

The demographics and clinical data of all patients are presented in Table 1. There were 140 patients with osteoporotic hip fracture recruited in this study, with 40 male patients $(28.6 \%)$. The mean age for males were $74.1 \pm 9.5$ years, while the mean age for females were $77.4 \pm 9.1$ years, and the difference was not statistically significant $(\mathrm{p}=0.06)$. The majority were non-smokers $(77.9 \%)$. 
Table 1

Demographic and clinical data of patients with osteoporotic hip fracture

Clinical characteristics

Number of female patients

Number of male

$\mathbf{P}$

(\%), $n=100$

patients(\%), $n=40$

value 


\begin{tabular}{|c|c|c|c|}
\hline Clinical characteristics & $\begin{array}{l}\text { Number of female patients } \\
(\%), n=100\end{array}$ & $\begin{array}{l}\text { Number of male } \\
\text { patients(\%), } n=40\end{array}$ & $\begin{array}{l}P \\
\text { value }\end{array}$ \\
\hline Number of patients & $100(71.4 \%)$ & $40(28.6)$ & $\mathrm{n} / \mathrm{a}$ \\
\hline Mean age & $77.4 \pm 9.1$ years & $74.1 \pm 9.1$ years & 0.06 \\
\hline Ethnicity & $58(58)$ & $22(55)$ & 0.49 \\
\hline Chinese & $21(21)$ & $5(12.5)$ & $<0.001$ \\
\hline Malay & $11(11)$ & $7(17.5)$ & 0.54 \\
\hline Iban & $9(9)$ & $6(15)$ & 0.69 \\
\hline Bidayuh & $1(1)$ & $0(0)$ & 0.22 \\
\hline Indian & $93(93)$ & $16(40)$ & $<0.001$ \\
\hline Smoking history & $6(6)$ & $18(45)$ & 0.55 \\
\hline Non smoker & $1(1)$ & $6(15)$ & \\
\hline Ex smoker & $65(65)$ & $26(65)$ & \\
\hline Current smoker & $54(54)$ & $24(60)$ & \\
\hline More than one co-morbidity & $45(45)$ & $16(40)$ & \\
\hline Type of hip fracture & $1(1)$ & $0(0)$ & \\
\hline Neck of femur & $21(21)$ & 15(37.5) & \\
\hline Intertrochanteric & $24(24)$ & $5(12.5)$ & \\
\hline Subtrochanteric & $19(19)$ & $7(17.5)$ & \\
\hline Management of hip fracture & $19(19)$ & $5(12.5)$ & \\
\hline Conservative management & $17(17)$ & $8(20)$ & \\
\hline \multirow{2}{*}{$\begin{array}{l}\text { Proximal femoral nail } \\
\text { antirotation (PFNA) }\end{array}$} & $13(13)$ & $20(50)$ & \\
\hline & $96(96)$ & $38(95)$ & \\
\hline \multicolumn{4}{|l|}{ Thompson hemiarthroplasty } \\
\hline \multicolumn{4}{|l|}{ Total hip replacement (THR) } \\
\hline \multicolumn{4}{|l|}{ Secondary osteoporosis } \\
\hline \multicolumn{4}{|l|}{ Outcome } \\
\hline \multicolumn{4}{|l|}{ Alive } \\
\hline Dead & & & \\
\hline
\end{tabular}


Most of the patients sustained neck of femur fracture(55.7\%) followed by intertrochanteric(43.6\%) and subtrochanteric $(0.7 \%)$. Surgical intervention was the mainstay of treatment in $74.2 \%$ while conservative treatment was administered in $25.7 \%$.

The secondary osteoporosis causes that were identified among all patients were COPD, hypogonadism, thyroid disorders, chronic kidney disease (CKD)/End stage renal failure (ESRF), glucocorticoid-induced osteoporosis (GIOP), rheumatoid arthritis, androgen deprivation therapy (ADT), asthma, early menopause, aromatase inhibitor therapy, malnutrition from previous gastrectomy, and prostate cancer. $50 \%$ of the male patients had identifiable cause of secondary osteoporosis, while only $13 \%$ of the female patients had secondary osteoporosis $(p<0.001)$. Table 2 shows the causes of secondary osteoporosis in the male osteoporotic hip fractures. The main causes of secondary osteoporosis in men were hypogonadism (30\%) followed by COPD, GIOP and CKD.

Table 2

Causes of secondary osteoporosis among male osteoporotic hip fractures

\begin{tabular}{|ll|}
\hline Causes of secondary osteoporosis, $\mathbf{n = 2 0}$ & Number of patients(\%) \\
\hline Hypogonadism & $6(30)$ \\
COPD & $4(20)$ \\
GIOP & $3(15)$ \\
CKD/ESRF & $3(15)$ \\
ADT & $1(5)$ \\
Thyroid disorders & $1(5)$ \\
Prostate cancer & $1(5)$ \\
Previous gastrectomy & $1(5)$ \\
\hline
\end{tabular}

During the inpatient and 3-month follow up period, 2 men and 4 women died. There was no statistical significance between the mortality rates between male patients $(5 \%)$ and female patients $(4 \%)(p=0.55)$.

\section{Discussion}

Even though we are facing an aging population with an expected rise in cases of osteoporotic hip fractures, there is still poor awareness regarding osteoporosis. Male osteoporosis, being less common than female osteoporosis, continues to receive little attention in terms of screening, diagnosis and treatment. This can hopefully be remedied by increased clinical data in the field of osteoporosis. There is increasing interest in the field of osteoporosis in Malaysia. The Malaysian clinical guidance for the management of osteoporosis included some data regarding the incidence and treatment for male osteoporosis $^{16}$. A study from Malaysia examined the age-related changes in bone health in men and women $>50$ years old using quantitative ultrasound (QUS), and the authors found significant age-related 
decline of bone health indices observed in females but not in males ${ }^{17}$. Yeap et al examined the depth and sources of osteoporosis knowledge among the public in Malaysia using a self-administered questionnaire distributed among attendees of health forums ${ }^{18}$. They found that $87.1 \%$ of attendees have heard of osteoporosis, and significantly more women than men $(p=0.015)$ have heard of osteoporosis. Data from other Asian countries are available as well, with the Asian Osteoporosis comparing hip fracture data from Hong Kong SAR, Singapore, Malaysia, and Thailand (Chiang Mai) in $1997^{19}$. The study reported the age-adjusted incidence rate for men and women as follows (per 100,000): Hong Kong 180 and 459, Singapore 164 and 442, Malaysia 88 and 218, Thailand 114 and 289; compared with US Whites 187 men and 535 women. In our study, the incidence of male osteoporotic hip fracture was $28.6 \%$, which was similar to other studies. In our study, as expected, the proportion of women with osteoporotic hip fractures outnumbered the men. Both groups have a mean age of $>70$ years. However, there was no statistically significant difference in mortality rates among the men and women in our study. This may be due to both groups being in the elderly group with co-morbidities.

Our current study showed that $50 \%$ of our male patients and $13 \%$ of female patients had secondary osteoporosis. This finding is consistent with reported data of $30 \%$ of post-menopausal women and 50 $80 \%$ of men were found to have secondary osteoporosis ${ }^{20}$. The osteoporosis treatment may differ for different underlying conditions, and certain conditions and medication prescription may prompt the clinician to consider bone health issues ${ }^{20}$. Ryan et al examined 234 men diagnosed with osteoporosis via DXA and measured 25-OH-vitamin D, testosterone, luteinizing hormone, follicular stimulating hormone (FSH), thyroid stimulating hormone (TSH), and spot urinary calcium-to-creatinine. $75 \%$ had secondary osteoporosis including hypogonadism, vitamin D deficiency, hypercalciuria, subclinical hyperthyroidism, and hyperparathyroidism ${ }^{21}$. The authors showed that a modest clinical and laboratory evaluation of osteoporotic men will provide useful information in identifying secondary osteoporosis. History, physical examination and basic laboratory investigation should be performed, and additional testing as appropriate. Colangelo et al proposed that after history and physical examination, a first-level laboratory test of full blood count, erythrocyte sedimentation rate (ESR), serum calcium, phosphorus, creatinine, alkaline phosphatase, total protein with electrophoresis and a 24-hour urinary calcium should be performed ${ }^{22}$. Following that, ionized calcium, parathyroid hormone (PTH), 25-OH-vitamin D, TSH, dexamethasone suppression test, serum and urinary immunofixation, anti-transglutamase antibodies, testosterone in men, serum tryptase and ferritin, should be considered clinically.

There were three men on glucocorticoids and one man on ADT in our study. Patients on these medications are recommended for osteoporosis evaluation (including Fracture Risk Assessment Tool (FRAX), BMD), calcium and vitamin D supplementation, and treatment with bisphosphonate, denosumab or teriparatide as appropriate ${ }^{22}$. Adler et al examined 115 men on ADT referred for DXA and found 33\% would need osteoporosis treatment ${ }^{23}$. More clinicians need to be made aware of osteoporosis evaluation when prescribing medications such as ADT and glucocorticoids. 
There has been much development in the treatment of post-menopausal osteoporosis, but data for male osteoporosis are notable as well. There is less evidence for treatment of male osteoporosis due to the smaller number of male participants in clinical trials. Evidence-based treatment for male osteoporosis are bisphosphonates (alendronate, risedronate, zoledronic acid), denosumab and teriparatide ${ }^{24-30}$. There is compelling evidence that current osteoporosis treatment is equally effective in men and women, not only to increase BMD but also to prevent osteoporotic fractures ${ }^{31}$. Effective treatment for GIOP for men and for male osteoporosis on ADT include bisphosphonates, denosumab and teriparatide ${ }^{28,32-33}$. Testosterone replacement is indicated for symptomatic hypogonadal men, but data on its efficacy for fracture prevention is lacking. Thus, additional osteoporosis treatment may be needed, especially in men with very low testosterone who are at high risk of bone loss and/or men not able to receive testosterone replacement ${ }^{34}$.

However there still exists a treatment care gap between men and women. The Canadian Multicentre Osteoporosis Study found that between 1996 until 2002, 90\% of men with fragility fractures remained undiagnosed and untreated for osteoporosis ${ }^{35}$. Yeap et al found that following a hip fracture, only $36.8 \%$ patients (men and women) received treatment, but out of these, $24.2 \%$ were on calcium and vitamin D only ${ }^{36}$.

There is a need to increase awareness of male osteoporosis among clinicians, so a diagnosis is made and appropriate treatment administered, especially among those with fragility fractures and those at risk of secondary osteoporosis. Other guidelines also recommend personalized assessment, obtaining DXA and FRAX in those at risk of osteoporosis, and starting appropriate treatment ${ }^{37-38}$. The Canadian Osteoporosis Society recommends screening men $>65$ years old for osteoporosis, while the National Osteoporosis Foundation and International Society for Clinical Densitometry and the Endocrine Society recommend screening all men $>70$ years old or men aged 50-69 years old with risk factors ${ }^{38}$. Alswat et al analyzed the rate of osteoporosis screening between men and women in primary care, and men had a screening rate of $18.4 \%$ compared to females screening rate of $60 \% 39$. De Martinis and colleagues also highlighted the gender bias in osteoporosis screening, and found that among those referred for osteoporosis screening at their center, $94.5 \%$ were women while only $5.4 \%$ were men. They also found that men were under-screened for osteoporosis, exhibit secondary osteoporosis more frequently, and had a higher calculated risk for hip fractures compared to women ${ }^{40}$.

The limitations in our study are:

1. The death outcome was collected at inpatient and at 3 month-follow up visit only. This may not reflect the one-year mortality rate.

2. Data collection was temporarily halted during the height of the Coronavirus Disease 2019 (COVID19) pandemic as the fracture liaison services was temporarily stopped. The number of cases in this study may not reflect the true incidence of male osteoporotic hip fractures. 
3. Some investigations results were not available for the secondary osteoporosis screening, and the number of secondary osteoporosis may not be truly reflected.

\section{Conclusion}

The gender difference in osteoporotic hip fractures in the elderly are the increased incidence women compared to men, and men having significantly increased incidence of secondary osteoporosis. There does not seem to be a difference of mortality rate between men and women in this study. Clinicians should be more aware of the importance of screening, diagnosis and treatment of osteoporosis especially in the context of an aging population.

\section{Abbreviations}

BMD: bone mineral density

SD: standard deviation

DXA: dual energy X-ray absorptiometry

CKD: chronic kidney disease

ESRF: end-stage renal failure

GIOP: Glucocorticoid-induced osteoporosis

ADT: androgen deprivation therapy

FRAX: Fracture risk assessment tool

\section{Declarations}

Availability of materials and data: the data is available from the corresponding author upon reasonable request

\section{Acknowledgements}

The authors would like to thank Dr Mohd Adam bin Bujang, Research Officer, Clinical Research Center, Sarawak General Hospital for his guidance and advice regarding the statistical analysis in this study.

We would like to thank the Director General of Health Malaysia for his permission to publish this article.

\section{Authors' contributions}

SAW is the first author, conceptualized the study, performed literature review, collected data, performed the statistical analysis and drafted the manuscript. TIK, CSL, CYK, BSMS, LKH, LWWH, TCL, TJK, AS 
collected data, and drafted the manuscript. ATJ provided DXA scan reports, collected data, reviewed the manuscript and is the corresponding author. All authors read and approved the final manuscript.

\section{Additional information}

\section{Competing interests}

The authors declare that they have no competing interests for this study.

\section{Funding}

No funding was received for this study.

\section{Ethics approval and consent to participate}

This study received ethics approval from the Malaysian Medical Research and Ethics Committee (MREC) (NMRR-19-323-46068 IIR). All participants in this study provided informed consent to participate.

\section{References}

1. Cheung, C. L. et al. An updated hip fracture projection in Asia: The Asian Federation of Osteoporosis Societies study.Osteoporosis and Sarcopenia.4, 16-21(2018)

2. Adler, R. A. Osteoporosis in men: a review.Bone Research. 2,14001(2014)

3. Walsh, J. S. \& Eastell, R. Osteoporosis in men.Nat Rev Endocrinol.9(11), 637-45(2013)

4. Willson, T. et al. The clinical epidemiology of male osteoporosis: a review of the recent literature.Clinical epidemiology.7,65-76(2015)

5. Cawthon, P. M. Gender differences in osteoporosis and fractures.Clin Orthop Relat Res.469,19001905(2011)

6. Haentjens, P. et al. Meta analysis: Excess mortality after hip fracture among older men and women.Ann Intern Med.152(6),380-390(2010)

7. Simunovic, N. et al. Effect of early surgery after hip fracture on mortality and complications: systematic review and meta-analysis.CMAJ;182(15),1609-1616(2010)

8. Gielen, E. et al. Osteoporosis in men.Best Practice \& Research Clinical Endocrinology \& Metabolism.25, 321-335(2011)

9. Cawthon, P. M. et al. Osteoporosis in men: Findings from the Osteoporotic Fractures in men study (MrOs).Ther Adv Musculoskel Dis.8(1), 15-27(2016)

10. Adler, R. A. Update on osteoporosis in men.Best Practice \& Research Clinical Endocrinology \& Metabolism.32(5), 759-772(2018)

11. Ebeling, P. R. Osteoporosis in Men.Curr Opin Rheumatol, 25, 542-552(2013)

12. Mohammad Anwar Hau Abdullah, Ahmad Tajuddin Abdullah (Eds). Annual Report of the NORMRegistry 2009. Kuala Lumpur, Malaysia: National Orthopaedic Registry Malaysia 2009 
13. Isnoni, I. et al. Pre-injury demographic patterns of patients sustaining hip fractures in Malaysia.Malaysian Orthopaedic Journal.6(4),11-15. (2012)

14. Sabarul, A. M. Current scenario of hip fracture cases in Malaysia.Research Updates in Medical Sciences (RUmeS). 3(3),1-2. (2015)

15. Mohamed, M. S. A. I. N. et al. Review of the risk factor of osteoporosis in the Malaysian population.Research Updates in Medical Services (RUmeS). 3(1),77-82. (2015)

16. Yeap, S. S. et al. A summary of the Malaysian Clinical Guidance on the management of postmenopausal and male osteoporosis, 2015.0steoporosis and Sarcopenia.2,1-12(2016)

17. Chin, K. Y. et al. Effects of age, sex and ethnicity on bone health status of the elderly in Kuala Lumpur, Malaysia.Clinical Interventions in Aging.11,767-773(2016)

18. Yeap, S. S., Goh, E. M. L. \& Gupta, E. D. Knowledge about osteoporosis in a Malaysian population.Asia-Pacific Journal of Public Health.22(2), 233-241(2010)

19. Lau, E. M. C. et al. The incidence of hip fracture in four Asian countries: The Asian osteoporosis study.Osteoporos Int.12,239-243(2001)

20. Mirza, F. \& Canalis, E. Secondary osteoporosis: pathophysiology and management.Eur J Endocrinol.173(3), R131-R151(2015)

21. Ryan, C. S., Petkov, V. I. \& Adler, R. A. Osteoporosis in men: the value of laboratory testing.Osteoporos Int. 22, 1845-1853(2011)

22. Colangelo, L. et al. Understanding and managing secondary osteoporosis. Expert Review of Endocrinology \& Metabolism.14(2), 111-122(2019)

23. Adler, R. A., Hastings, F. W. \& Petkov, V. I. Treatment thresholds for osteoporosis in men on androgen deprivation therapy: T score vs FRAX.Osteoporos Int.21,647-653(2010)

24. Khan, A. A. et al. Management of osteoporosis in men: an update and case example.CMAJ.176(3), 345-8(2007)

25. Giusti, A. \& Bianchi, G. Treatment of primary osteoporosis in men.Clinical Interventions in Aging.10,105-115(2015)

26. Kaufman, J. M. et al. Treatment of osteoporosis in men.Bone,134-144(2013)

27. Korpi-Steiner, N., Milhorn, D. \& Hammet-Stabler, C. Osteoporosis in men. Clin Biochem. 47(10-11), 950-959 (2014)

28. Watts, N. B. et al. Osteoporosis in men: An Endocrine Society Clinical Practice Guideline.J Clin Endocrinol Metab. 97, 1802-1822(2012)

29. Zeng, L. F. et al. Does routine anti-osteoporosis medication lower the risk of fractures in male subjects? An updated systematic review with meta analysis of clinical trials.Front Pharmcol.10, 882(2019)

30. Porcelli, T. et al. Male osteoporosis: diagnosis and management- should the treatment and the target be the same as for female osteoporosis?European Journal of Endocrinology.183, R75-R93(2020) 
31. Laurent, M. et al. Osteoporosis in older men: Recent advances in pathophysiology and treatment.Best pract Res Clin Endocrinol Metab.27(4),527-39(2013)

32. Glüer, C. C. et al. Comparative effects of teriparatide and risedronate in glucocorticoid-induced osteoporosis in men: 18-month results of the EuroGIOPs trial.Journal of Bone and Mineral Research. 28(6),1355-1368(2013)

33. Mosekilde, L., Vestergaard, P. \& Rejnmark, L. The pathogenesis, treatment and prevention of osteoporosis in men.Drugs. 73,15-29(2013)

34. Rochira, V., Antonio, L. \& Vanderschueren, D. EAA clinical guideline on management of bone health in the andrological outpatient clinic.Andrology.6,272-285(2018)

35. Papaioannou, A. et al. The osteoporosis care gap in men with fragility fractures: the Canadian Multicentre Osteoporosis Study.Osteoporos Int.19,581-587(2008)

36. Yeap, S. S. et al. Trends in post osteoporotic hip fracture care from 2010 to 2014 in a private hospital in Malaysia.Osteoporosis and Sarcopenia.3(2),112-116(2017)

37. Qaseem, A. et al. Screening for osteoporosis in men: A clinical practice guideline from the American College of Physicians.Ann Int Med.148, 680-684(2008)

38. Alswat, K. A. Gender disparities in osteoporosis.J Clin Med Res.9(5), 382-387(2017)

39. Alswat, K. \& Myers Adler, S. Gender differences in osteoporosis screening: retrospective analysis.Arch Osteoporos.7,311-313(2012)

40. De Martinis, M. et al. Gender difference in osteoporosis: a single center observational study. World J Mens Health.39(4), 750-759(2021) 